\title{
Closure of an Open Wound Associated with Bisphosphonate-Related Osteonecrosis of the Jaw in a Breast Cancer Patient
}

\author{
Nafiseh Soolari ${ }^{1}$ and Ahmad Soolari ${ }^{2}{ }_{*}$ \\ ${ }^{I}$ Department of Communication, College of Arts and Humanities, University of Maryland, College Park, MD, USA \\ ${ }^{2}$ Private Practice in Periodontology, Silver Spring and Potomac, MD, USA
}

\begin{abstract}
Background and Objective: Many clinicians will not treat patients presenting with bisphosphonate-related osteonecrosis of the jaw following long-term use of bisphosphonates because of the lack of predictable outcomes.

Materical and Methods: The patient presented with pain from a nonhealing lesion in the posterior maxilla following extraction of the maxillary right third molar. The lesion had not responded to any conventional dental treatment. The patient had suffered from breast cancer, and her treatment included several years of therapy with Zometa (zoledronic acid), a bisphosphonate.

Results: The patient stopped taking Zometa and commenced rinsing with phosphate buffer-stabilized $0.1 \%$ chlorine dioxide-containing mouthwash. After 5 months, changes in the morphology of the lesion were noted and the soft tissue had closed over the open wound.

Conclusion: Cessation of bisphosphonate therapy and usage of a phosphate buffer-stabilized $0.1 \%$ chlorine dioxide-containing mouthwash lessened the patient's pain and resulted in closure of the soft tissue lesion.
\end{abstract}

Keywords: Bisphosphonate, Zometa, Osteonecrosis of the Jaw, Breast cancer, Cancer.

\section{INTRODUCTION}

Breast cancer is a frightening condition for women, although it is one of the most treatable cancers if detected early. Breast cancers occur as a result of genetic abnormalities (e.g., mutations of the BRCA1 and BRCA2 genes) that happen as a result of the aging process and life in general $[1$, 2]. However, other factors also play a role in breast cancer risk, including the presence of a first-degree relative who has had breast cancer. Exposure to estrogen and progesterone can also increase the risk of breast cancer somewhat, depending on a woman's lifetime exposure, the types of hormones used (beyond those that occur naturally), and the method of administration [3].

Surgery is the primary treatment for breast cancer, although it may be combined with chemotherapy, hormone therapy, and radiation therapy. Because some breast cancer treatments can cause bone loss (osteoporosis), many women being treated for breast cancer are also prescribed a bisphosphonate (BP). Some BPs, in addition to helping slow bone loss, can also help treat breast cancer; Raloxifene, a drug frequently used to reduce osteoporosis in women, has been shown to be effective in reducing invasive breast cancer by about 50\% [4]. However, treatment with BPs is not without side effects, and BP-related osteonecrosis of the jaw

*Address correspondence to this author at the 11616 Toulone Drive. Potomac, MD 20854, USA; Tel: 301 674-9815; Fax: 240-845-1087;

E-mail: asoolari@gmail.com
(BRONJ) has been reported frequently. Researchers believe that BRONJ may develop because BPs stop the body from repairing microscopic damage to the jawbone that can happen during routine dental procedures or from everyday wear and tear. However, it is still not clear why ONJ occurs in some people and not in others [1]. Breast cancer patients with a history of long-term use of intravenous BPs are at risk for complications related to ONJ. Tooth extraction has been reported as one of the major precipitating factors for ONJ [5]. Other contributing factors, such as radiation therapy and invasive surgical procedures, have also been discussed [6].

Reports of ONJ have become increasingly common since 2003 [7-10], and the incidence of jaw necrosis in cancer patients who are on BPs is approximately $6 \%$ to $7 \%[5,6,11$ 14]. Many cases of ONJ have been described; individual authors reported $63,17,10$, and 10 cases of ONJ, respectively $[5,6,8,11]$. However, treatment of ONJ is problematic and unpredictable. Therefore, the authors report here on their successful treatment of a patient who developed BRONJ following extraction of the maxillary right third molar, which left a nonhealing region in the posterior maxilla. The patient had been on long-term intravenous BPs and presented with a chief complaint of aches and pain along with a "sticky, paint-like smell" in the mouth.

\section{PATIENT REPORT}

The patient was a 38-year-old woman from South Asia who had been diagnosed with breast cancer in 1997. She had moved to the United States in 2000. Consultation with the patient's oncologist revealed that the patient had been on a 


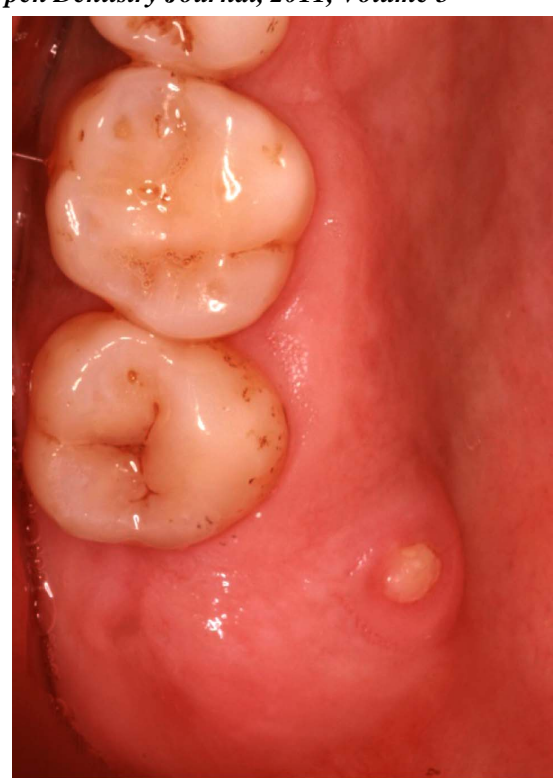

Fig. (1). BRONJ lesion that developed following tooth extraction.

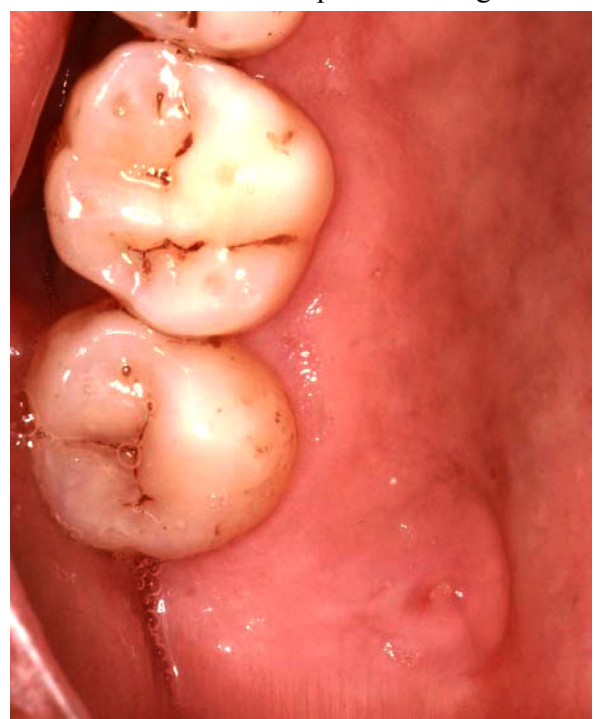

Fig, (2). Wound closure after 5 months of treatment with PBSCD mouthwash.

monthly protocol of $4 \mathrm{mg}$ of zoledronic acid (Zometa) intravenously from 2002 until 2009, when the maxillary right third molar was extracted. Three months after the extraction, the patient complained of aches and pain in the posterior right maxilla. Clinical oral evaluation revealed a $3 \times 3 \times 2$ $\mathrm{mm}$ BRONJ lesion (Fig. 1) associated with the palatal aspect of the area of the extracted tooth. The patient was treated with amoxicillin clavulanate $(500 \mathrm{mg}$ orally three times per day for 10 days) and chlorhexidine rinse $(0.12 \%$ twice daily for 30 seconds). The patient responded positively and stated that she felt better. The aching disappeared, but the open wound remained and was plaque retentive and a nuisance to the patient.

Consultation with the patient's oncologist resulted in discontinuation of Zometa treatment. The authors then instructed the patient to rinse with phosphate buffer-stabilized $0.1 \%$ chlorine dioxide-containing (PBSCD) mouthwash (CloSYS II, Rowpar Pharmaceuticals) for 30 seconds three times a day. Five months later, the patient presented with almost complete soft tissue healing over the previously exposed necrotic bone (Fig. 2). The patient was happy with the mouthwash and stated that the rinse helped her to feel better about the area.

\section{DISCUSSION}

Patients with breast cancer are often treated intravenously with zoledronic acid or other BPs to suppress osteoclast activity. As many studies have suggested, patients who receive intravenous BP therapy along with additional chemotherapy and corticosteroids are at a high risk for ONJ [15]. The present patient received zoledronic acid on a monthly basis from 2002 to 2009 . The discontinuation of zoledronic acid treatment in January 2009 appeared to help, in contrast to the findings of Ruggiero et al, who stated that "there is no evidence to suggest interrupting bisphosphonate therapy will prevent or lower the risk of ONJ" [8]. However, since the half-lives of various BPs have been reported to range from 147 hours (in plasma levels [16]) to a very long 10 years (in bone [17]), it is possible that cessation may help in such cases.

The intravenous BP used in the present patient were of a nitrogen-based nature [18] and is commonly known as zoledronic acid [18-20]. Zoledronic acid and other nitrogenbased BPs, such as pamidronate [21], possess a scaffold, which presents with a nitrogen motif as one of its side chains [18]. This allows these drugs to work as better antagonists of bone resorption than their non-nitrogen-containing counterparts $[18,20]$. However, long-term use of zoledronic acid has a cumulative risk factor for ONJ that climbs with an increase in infusion levels-from $6.7 \%$ after 20 treatments to $31.7 \%$ after escalation to 36 treatments [22]. The likelihood of zoledronic acid to produce ONJ alone was also compared to that of other BPs, and a 9.5-fold greater risk has been reported [23]. Median time to onset of ONJ is also shorter for zoledronic acid than for pamidronate: 12 months versus 24 months, respectively [20, 24].

Patients who have received oral BP treatment may also be at risk for developing ONJ but to a lesser degree than their intravenously treated counterparts $[8,25,26]$. Rose et al stated that, although "strong clinical research is lacking," it remains true that patients taking oral BPs remain at risk of ONJ if their therapy exceeds 3 years [26]. Ruggiero concurred $[7,8]$, and these experts suggest that although the risk is rare, it is necessary for those patients who have endured oral BP therapy for at least 3 years to receive the same precautionary treatments as their intravenously treated counterparts. Rose et al also stressed the importance of coordination between a patient's health care providers to prevent complications related to any type of dental treatment-surgical or otherwise $[8,26]$.

Because BRONJ has been reported extensively since 2003 [7-10], the question should be asked of whether the potential risk of harming the jawbone is balanced by the reward of the stronger skeleton overall provided by BP treatment [27]. During a minisymposium on BPs and ONJ held in 2006, Somerman and McCauley suggested several reasons why the jaws are affected [27]. First, unlike long bones and vertebrae, the maxilla and mandible are formed primarily via intramembranous bone formation $[6,27]$ and second, in con- 
trast to the red marrow found in long bones, ribs, and vertebrae, the jaw contains fatty marrow and localized hematopoietic marrow, which is found in adults $[10,11,28]$. The presence of a hematopoietic environment in the skeleton is likely protective in the healing response of the bone-thus, the absence thereof in the oral cavity may be a predisposing factor to complications $[6,12,13,27]$. This, in addition to the notion that the bones of the jaw are continuously exposed to microorganisms, may explain the ineffectiveness of an antibiotic in reaching its target to manage a non healing region of ONJ. Somerman and McCauley also noted the sensitivity of oral tissues to alterations in phosphate and pyrophosphate levels, as well as to changes in the phosphate/pyrophosphate ratio, as another predisposing factor for emerging ONJ. In addition to what has already been mentioned, the authors suggested that BPs may play a role in reducing vascularity of the target tissue, such as the jaw, because of the effect BPs have on osteoclasts $[9,10,13,14$, 27, 29].

As one of the major precipitating factors for ONJ, extraction has been reported to be associated with the onset of exposed ONJ lesions in $70 \%[5,29]$ to $100 \%$ of cases [30]. Other factors, such as radiation therapy and invasive surgical procedures [6], have been noted as predisposing influences as well. Newer information indicates that the etiology of ONJ is likely multifactorial [15, 31, 32], including but not limited to corticosteroids, chemotherapy, radiotherapy, jaw trauma, denture trauma, infection, and cancer $[7,8,17,33$, 34].

Periodontal disease was found to be the most common dental comorbidity in cases of BRONJ [35]. In 2005 the American Academy of Periodontology classified ONJ risk factors into "definite," "probable," and "additional" risk factor categories [36]. Those that are considered "definite" risk factors for ONJ are major trauma to the jawbone area, fractures, dislocations, sickle cell disease, Caisson disease, Gaucher disease, irradiation, chemotherapy, and arterial disease. ONJ risk factors classified as "probable" deal primarily with corticosteroids, alcohol consumption, smoking habits, lupus, kidney disease, liver disease, lipid disturbances, connective tissue diseases, and pancreatitis. "Additional" risk factors include categories such as a history of cancer, gender (women are more predisposed), advanced age, the presence of edentulous regions, surgical dental procedures, prior infection of the jaw area, combined cancer therapy, blood dyscrasias, anemia, and coagulopathy [36].

Sedghizadeh et al. [37] explored the role of biofilm and hypothesized that the pathogenesis of ONJ may represent a biofilm-mediated infectious disease in the context of BP therapy and that therefore a potential target for therapy should include antibiofilm modalities [37]. Grootvelt et al. presented evidence for the microbicidal activity of a chlorine dioxide-containing oral rinse that demonstrated the ability of the oral rinse to diminish salivary levels of Streptococcus mutans and lactobacilli [38]. Its positive impact on malodor was investigated recently by Shinada et al. [39]. The authors concluded that rinsing with chlorine dioxide-containing oral rinse reduced malodor significantly. Moreover, the mouthwash appeared to be effective in reducing plaque, the accumulation of tongue coating, and counts of Fusobacterium nucleatum in saliva [39]. The aforementioned studies suggest that the inhibitory effect of chlorine dioxide-containing mouthwash on harmful oral bacteria may play a positive role in sequestration of necrotic bone, wound healing, and resolution of BRONJ.

In the present situation, it is not clear when and how the necrotic bone was sequestered from the area, because the patient did not state that she had lost bone from her palate. The authors speculate that sequestration took place, but because of the small size of the lesion, the patient could not feel it or detect it. Although, as mentioned earlier, there is disagreement regarding whether cessation of BPs may help to prevent ONJ [8], it can be speculated that in this case a combination of discontinuation of zoledronic acid and initiation of the rinsing protocol with PBSCD helped resolve the non healing lesion. The authors are in agreement with Soolari et al [40] and Marder and Marder's suggestion that PBSCD mouthwash has anti-inflammatory and antifungal activities and has the potential to facilitate release of necrotic bone and closure of an open wound [41].

On a positive note, a recent study observed favorable results in the treatment of BP-associated osteonecrosis with a combination of marginal resection and platelet-derived growth factors, with most patients showing complete wound healing [35]. However, treatment and supportive therapy of a non healing lesion for patients suffering from BRONJ continue to present a major challenge to health care providers. The present protocol represents a noninvasive treatment option for patients with BRONJ.

\section{RECOMMENDATIONS}

Through continued cooperation between healthcare providers, dental surveillance, prophylactic care, and most important, early diagnosis and management, morbidity resulting from ONJ caused by BP use may be reduced greatly or even eliminated in the future [21].

Although there is currently no proven effective treatment for BRONJ lesions, the authors recommend the following.

1) Close coordination is essential between medical and dental practitioners for patients who are starting BP therapy, those who have started BPs but not developed ONJ, and patients who have already developed ONJ [21]. All invasive dental procedures should be completed well in advance of the initiation of intravenous BP treatment, with continued dental supervision thereafter. Patients at the highest risk are cancer patients who are receiving a combination of chemotherapy and corticosteroid therapy, long-term users of BP (particularly zoledronic acid), removable partial denture users, and patients with poor oral hygiene and periodontal disease [21].

2) The use of an alternate infusion schedule for zoledronic acid is recommended to reduce the risk of ONJ in patients with breast cancer. Treatments should be conducted monthly during the first year and every 3 months thereafter, rather than on the standard schedule, in which infusions are received on a monthly basis for an indeterminate period [34]. 
3) In addition, if ONJ has already emerged, antibiotics should be prescribed before and after any pending dental procedure, along with a chlorhexidine rinse, to prevent complications or further progression of the ONJ lesion $[7,8,26]$.

4) A fixed partial denture is recommended over a removal partial denture to reduce the likelihood of the emergence of ONJ by denture-induced trauma [8].

5) Frequent dental prophylaxis is advised to eliminate plaque from the necrotic region and to reduce antigen loads in general [21].

\section{ACKNOWLEDGMENT}

The authors, in reference to this case report, have no affiliation with or financial support from any institution in the completion of this study.

\section{CONFLICT OF INTEREST}

There are no conflicts of interest and no financial relationships related to any products involved in this study.

\section{REFERENCES}

[1] Breastcancer.org: Breast cancer, 2010. Available: http://www.breastcancer.org. (Accessed 2011).

[2] U.S. National Library of Medicine. Genetics home reference: Breast cancer 2007. Available: http://ghr.nlm.nih.gov/condition=breastcancer. (Accessed 2011).

[3] ESHRE Capri Workshop Group. Hormones and breast cancer. Hum Reprod 2004; 10(4): 281-3.

[4] National Cancer Institute. Results of the study of tamoxifen and raloxifene (STAR) released: osteoporosis drug raloxifene shown to be as effective as tamoxifen in preventing invasive breast cancer. Available: http://www.cancer.gov/newscenter/pressreleases/STARresultsApr172006 . 2006; (Accessed 2011).

[5] Bagan JV. Avascular jaw osteonecrosis in association with cancer chemotherapy: series of 10 cases. J Oral Pathol Med 2005; 34(2): 120-3.

[6] Migliorati CA. Bisphosphonates and oral cavity avascular bone necrosis. J Clin Oncol 2003; 21(22): 4253-4.

[7] Ruggiero SL. Osteonecrosis of the jaws associated with the use of bisphosphonates: a review of 63 cases. J Oral Maxillofac Surg 2004; 62(5): 527-34.

[8] Ruggiero SL. Practical guidelines for the prevention, diagnosis and treatment of osteonecrosis of the jaw in patients with cancer. J Oncol Pract 2006; 2(1):7-14.

[9] Marx RE. Pamidronate (Aredia) and zoledronate (Zometa) induced avascular necrosis of the jaws: a growing epidemic. J Oral Maxillofac Surg 2003; 61(9): 1115-7.

[10] Marx RE. Bisphosphonate-induced exposed bone (osteonecrosis/osteopetrosis) of the jaws: risk factors, recognition, prevention, and treatment. J Oral Maxillofac Surg 2005; 63(11): 1567-75.

[11] Migliorati CA. Managing the care of patients with bisphosphonateassociated osteonecrosis: an american academy of oral medicine position paper. J Am Dent Assoc 2005; 136(12): 1658-68.

[12] Migliorati CA, Siegel MA, Elting LS. Bisphosphonate-associated osteonecrosis: a long-term complication of bisphosphonate treatment. Lancet Oncol 2006; 7(6): 508-14.

[13] Woo SB. Osteonecrosis of the jaw and bisphosphonates. N Engl J Med 2005; 353(1): 99-102.

[14] Gralow J. Evolving role of bisphosphonates in women undergoing treatment for localized and advanced breast cancer. Clin Breast Cancer 2005; 5(Suppl 2): S54-62.

[15] Piesold JU, Al-Nawas B, Grötz KA. Osteonecrosis of the jaws by long term therapy with bisphosphonates. Mund Kiefer Gesichtschir 2006; 10(5): 287-300.
[16] Chen T, Berenson J, Vescio R, et al. Pharmacokinetics and pharmacodynamics of zoledronic acid in cancer patients with bone metastases. J Clin Pharmacol 2002; 42: 1228-36.

[17] Thorpe J. Alendronate (Fosamax) and risedronate (Actonel) revisited. Obstet Gynecol 2005; 106(2):402-4.

[18] Bilezikian JP. Osteonecrosis of the jaw: do bisphosphonates pose a risk? N Engl J Med 2006; 355(22): 2278-81.

[19] Dannemann C, Grätz KW, Riener MO, Zwahlen RA. Jaw osteonecrosis related to bisphosphonate therapy: a severe secondary disorder. Bone 2007; 40(4): 828-34.

[20] Zarychanski R. Osteonecrosis of the jaw associated with pamidronate therapy. Am J Hematol 2006; 81(1): 73-5.

[21] Weitzman R, Sauter N, Eriksen E, et al. Critical review: updated recommendations for the prevention, diagnosis, and treatment of osteonecrosis of the jaw in cancer patients. Crit Rev Oncol Hematol 2007; 62(2): 148-52.

[22] Calvo-Villas JM. Osteonecrosis of the jaw in patients with multiple myeloma during and after treatment with zoledronic acid. Med Clin (Barc) 2006; 127(15): 576-9.

[23] Zervas K, Verrou E, Teleioudis Z, et al. Incidence, risk factors and management of osteonecrosis of the jaw in patients with multiple myeloma: a single-centre experience in 303 patients. Br J Haematol 2006; 134(6): 620-3.

[24] Mavrokokki T, Cheng A, Stein B, Goss A. Nature and frequency of bisphosphonate-associated osteonecrosis of the jaws in Australia. $\mathrm{J}$ Oral Maxillofac Surg 2007; 65(3): 415-23.

[25] Bamias A. Osteonecrosis of the jaw in cancer after treatment with bisphosphonates: incidence and risk factors. J Clin Oncol 2005; 23(34): 8580-7.

[26] Rose L, Ruggiero S, Silverstein L. When can patients who are being treated with oral and IV bisphosphonates receive periodontal, implant, and/or oral surgical care? Inside Dent 2007; 3(9): 44-5.

[27] Somerman MJ, McCauley LK. Bisphosphonates: sacrificing the jaw to save the skeleton? IBMS BoneKey 2006; 3(9): 12-5.

[28] Krueger CD, West PM, Sargent M, Lodolce AE, Pickard AS. Bisphosphonate-induced osteonecrosis of the jaw. Ann Pharmacother 2007; 41(2): 276-84.

[29] Bagan JV, Jimenez Y, Murillo J, et al. Jaw osteonecrosis associated with bisphosphonates: multiple exposed areas and its relationship to teeth extractions. study of 20 cases. Oral Oncol 2006; 42(3): 327-9.

[30] Woo SB, Hellstein JW, Kalmar JR. Narrative review: bisphosphonates and osteonecrosis of the jaws. Ann Intern Med 2006;144(10): 753-61.

[31] Farrugia MC, Summerlin DJ, Krowiak E, et al. Osteonecrosis of the mandible or maxilla associated with the use of new generation bisphosphonates. Laryngoscope 2006; 116: 115-20.

[32] Wang H, Weber D, McCauley LK. Effect of long-term oral bisphosphonate on implant wound healing: literature review and a case report. J Orofac Pain 2007; 78: 584-94.

[33] Khamaisi M, Regev E, Yarom N, et al. Possible association between diabetes and bisphosphonate-related jaw osteonecrosis. J Clin Endocrinol Metab 2007; 92(3): 1172-5.

[34] Corso A, Varettoni M, Zappasodi P, et al. Different schedule of zoledronic acid can reduce the risk of the osteonecrosis of the jaw in patients with multiple myeloma. Leukemia 2007; 21(7): 1545-8.

[35] Adornato MC, Morcos I, Rozanski J. The treatment of bisphosphonate-associated osteonecrosis of the jaws with bone resection and autologous platelet-derived growth factors. J Am Dent Assoc 2007; 138(7): 971-7.

[36] American Academy of Periodontology. AAP Statement on Bisphosphonates, 2010. Available: www.perio.org/resourcesproducts/bisphosphonates.htm. (Accessed 20 March 2011).

[37] Sedghizadeh PP, Kumar SK, Gorur A, Schaudinn C, Shuler CF, Costerton JW. Microbial biofilms in osteomyelitis of the jaw and osteonecrosis of the jaw secondary to bisphosphonate therapy. J Am Dent Assoc 2009; 140(10): 1259-65.

[38] Grootvelt M, Silwood C, Gill D, Lynch E. Evidence for the microbicidal activity of a chlorine dioxide-containing oral rinse formulation in vivo. J Clin Dent 2001; 12(3): 67-70.

[39] Shinada K, Ueno M, Konishi C, et al. Effects of a mouthwash with chlorine dioxide on oral malodor and salivary bacteria: a randomized placebo-controlled 7-day trial. Trials 2010; 11: 14.

[40] Soolari E, Soolari A, Soolari A. Phosphate buffer-stabilized 0.1\% chlorine dioxide-containing mouth wash facilitate sequestration of bisphosphonate related osteonecrosis of jaw (BRONJ) lesion from 
a patient who presented with osteonecrosis of the jaw and a history of intravenous bisphosphonate use: case report. Translation Biomed 2010; 1(1): 7
[41] Marder MZ, Marder RW. Bisphosphonate-associated osteonecrosis experiences in a private practice. Dent Today 2008; 27(10): 99103.

Received: June 29, 2011

(C) Soolari and Soolari; Licensee Bentham Open.

This is an open access article licensed under the terms of the Creative Commons Attribution Non-Commercial License (http://creativecommons.org/licenses/by-nc/3.0/) which permits unrestricted, non-commercial use, distribution and reproduction in any medium, provided the work is properly cited. 